\title{
Brain-Derived Neurotrophic Factor and TrkB Modulate Visual Experience-Dependent Refinement of Neuronal Pathways in Retina
}

\author{
Xiaorong Liu, ${ }^{1,2,3}$ Ruslan N. Grishanin, ${ }^{2,3}$ Ravi J. Tolwani, ${ }^{5}$ René C. Rentería, ${ }^{1,2,3}$ Baoji Xu, ${ }^{6}$ Louis F. Reichardt, ${ }^{2,3,4}$ and \\ David R. Copenhagen ${ }^{1,2,3}$ \\ ${ }^{1}$ Department of Ophthalmology, ${ }^{2}$ Department of Physiology, ${ }^{3}$ Program in Neuroscience, and ${ }^{4}$ Howard Hughes Medical Institute, University of California, \\ San Francisco, San Francisco, California 94143, 5 Department of Comparative Medicine, Stanford University School of Medicine, Stanford, California 94305, \\ and ${ }^{\circ}$ Department of Pharmacology, Georgetown University Medical School, Washington, DC 20057
}

Sensory experience refines neuronal structure and functionality. The visual system has proved to be a productive model system to study this plasticity. In the neonatal retina, the dendritic arbors of a large proportion of ganglion cells are diffuse in the inner plexiform layer. With maturation, many of these arbors become monolaminated. Visual deprivation suppresses this remodeling. Little is known of the molecular mechanisms controlling maturational and experience-dependent refinement. Here, we tested the hypothesis that brainderived neurotrophic factor (BDNF), which is known to regulate dendritic branching and synaptic function in the brain, modulates the developmental and visual experience-dependent refinement of retinal ganglion cells. We used a transgenic mouse line, in which a small number of ganglion cells were labeled with yellow fluorescence protein, to delineate their dendritic structure in vivo. We found that transgenic overexpression of BDNF accelerated the laminar refinement of ganglion cell dendrites, whereas decreased TrkB expression or retina-specific deletion of TrkB, the cognate receptor for BDNF, retarded it. BDNF-TrkB signaling regulated the maturational formation of new branches in $\mathrm{ON}$ but not the bilaminated $\mathrm{ON}-\mathrm{OFF}$ ganglion cells. Furthermore, BDNF overexpression overrides the requirement for visual inputs to stimulate laminar refinement and dendritic branching of ganglion cells. These experiments reveal a previously unrecognized action of BDNF and TrkB in controlling cell-specific, experience-dependent remodeling of neuronal structures in the visual system.

Key words: retinal ganglion cell; RGC; brain-derived neurotrophic factor; BDNF; TrkB receptor; dendritic refinement; activity-dependent plasticity; transgenic/conditional knock-out mice

\section{Introduction}

The complex yet characteristic morphology of dendritic arbors defines the inputs a neuron receives. In retina, the distinctive laminar patterns of retinal ganglion cell (RGC) dendrites govern the responsiveness of RGCs to visual stimulation (Sernagor et al., 2001). ON RGCs, which respond to light increment, have monolaminated dendritic arbors in the inner region (sublamina $b$ ) of the inner plexiform layer (IPL), where they receive exclusive excitatory synaptic inputs from ON bipolar cells (Famiglietti and Kolb, 1976; Nelson et al., 1978). OFF RGC dendrites, also monolaminated, colocalize with OFF bipolar axonal terminals in sub-

Received Feb. 20, 2007; revised May 25, 2007; accepted May 26, 2007.

This work was supported by a Knights Templar Research Grant (X.L.), That Man May See, Research to Prevent Blindness (D.R.C.), National Institutes of Health grants (D.R.C., L.F.R.), and Howard Hughes Medical Institute (L.F.R.) We thank Drs. Jianhua Cang and Juliette Johnson for suggestions and comments. We also thank Sushama Varma for help in breeding BDNF-OE mice, Leah M. McGuire for RGC tracings, Tony Tran and Elizabeth Hawkes for genotyping, Haidong Yang for histology, and the University of California, San Francisco, Biomolecular Resource Center for performing the real-time $P C R$.

Correspondence should be addressed to David R. Copenhagen, Department of Ophthalmology, University of California, San Francisco, School of Medicine, 10 Koret Way, Room K140, San Francisco, CA 94143-0730. E-mail: cope@phy.ucsf.edu.

R. J. Tolwani's present address: Rockerfeller University, 1230 York Avenue, New York, NY 10021.

DOI:10.1523/JNEUROSCI.0779-07.2007

Copyright $\odot 2007$ Society for Neuroscience $\quad$ 0270-6474/07/277256-12\$15.00/0 lamina $a$ (Famiglietti and Kolb, 1976; Nelson et al., 1978). ONOFF RGCs respond to light onset and offset and have bilaminated dendrites arborizing in both sublaminas $a$ and $b$ of the IPL (Amthor et al., 1984). Early in postnatal development, the retina is populated with a preponderance of RGCs having bilaminated dendritic arbors (Maslim and Stone, 1988; Bodnarenko et al., 1999; Tian and Copenhagen, 2003; Diao et al., 2004). In mouse, the population of bilaminated RGCs is reduced significantly 2 weeks after eye opening. Furthermore, the developmental progression of bilaminated RGCs into monolaminated RGCs relies on visual experience. Dark rearing retards refinement (Tian and Copenhagen, 2003), and enrichment of the physical environment afforded mothers and their pups accelerates laminar refinement (Landi et al., 2007).

Brain-derived neurotrophic factor (BDNF), a neurotrophin family member, acts via its high-affinity receptor, TrkB, to regulate activity-dependent axonal and dendritic branching as well as synaptic and neuronal network formation in the CNS (Huang and Reichardt, 2001). Previous studies reveal mixed actions of $\mathrm{BDNF} /$ TrkB on dendritic growth or patterning of neurons in the CNS (Horch et al., 1999; Xu et al., 2000; Horch and Katz, 2002; Tolwani et al., 2002), making it difficult to predict a priori how BDNF and TrkB will affect neuronal structure in any given region 
of the nervous system. In the visual system, exogenous BDNF injected into the Xenopus retina reduced RGC dendritic arborization (Lom and Cohen-Cory, 1999). In contrast, BDNF induced dendritic growth in cultured dissociated rat RGCs (Bosco and Linden, 1999). However, in mouse retina, intraocular injections of BDNF antisense oligonucleotides blocked the accelerated refinement induced by enriched environments (Landi et al., 2007). Despite observed actions of exogenous BDNF on neuronal morphology in brain or retina, very few studies have examined the role of BDNF and TrkB signaling on dendritic refinement of identified cell types in an in vivo system.

Given that (1) BDNF and TrkB are expressed in the mouse retina (Bennett et al., 1999), (2) visual deprivation reduces levels of retinal BDNF (Seki et al., 2003; Mandolesi et al., 2005; Landi et al., 2007), and (3) BDNF activation of TrkB can regulate neuronal architecture in diverse types of neurons (McAllister et al., 1999; Poo, 2001), we tested the hypothesis that activation of TrkB by BDNF plays a role in maturational and visual experiencedependent refinement of RGC dendrites. This study combined molecular and anatomical analyses of genetically modified mice. We demonstrate that BDNF/TrkB signaling regulates both laminar refinement and dendritic branching of RGCs during postnatal development. These results establish a strong link between activity-induced remodeling of RGC dendrites and BDNF/TrkB signaling in mammalian retina.

\section{Materials and Methods}

Animals. Thy-1-yellow fluorescent protein (YFP) transgenic mice (line H; The Jackson Laboratory, Bar Harbor, ME) (Feng et al., 2000) were used to examine dendritic structures of RGCs. Transgenic mice overexpressing BDNF with a C-terminal myc-epitope tag under the control of the human $\beta$-actin promoter (BDNF-OE) were provided by Regeneron Pharmaceuticals (Tarrytown, NY) (Croll et al., 1999). Transgenic mice with reduced TrkB expression ( $\operatorname{TrkB}^{\mathrm{fBZ}}$ ) were created by inserting a rat TrkB cDNA flanked by loxP sites and followed by an SV40 (simian virus 40) poly(A) sequence into the first coding exon (Xu et al., 2000). TrkB ${ }^{\mathrm{fBZ}}$ mice did not exhibit a complete deletion of TrkB. Instead, TrkB expression was reduced to $25-30 \%$ of wild-type (WT) levels. Another strain of mice having a deletion of $\operatorname{trkB}$ gene in the retina $\left(\operatorname{Trk} B^{f / f}\right.$, Six3-Cre/+) were generated using Cre-mediated recombination. Mice heterozygous for $\operatorname{TrkB} B^{f /+}$ were crossed with Six3-Cre transgenic mice (Furuta et al., 2000) to generate mice heterozygous for both $T r k B^{f /+}$ and Six3-Cre $\left(\operatorname{TrkB} B^{f /+}\right.$, Six3-Cre/+). Mice heterozygous for the TrkB floxed allele $\left(\operatorname{Trk} B^{f /+}\right)$ and mice heterozygous for both TrkB floxed allele and Six3-Cre $\left(\mathrm{TrkB}^{f /+}\right.$, Six3-Cre/+) were crossed to generate the conditional trkB knock-outs (TrkB ${ }^{f / f}$, Six3-Cre/+). The conditional mutants are healthy and indistinguishable from their wild-type littermate controls. BDNFOE, TrkB hypomorphic mice, and $\operatorname{TrkB} B^{f /+}$, Six3-Cre/+ heterozygous mice were crossed with Thy-1-YFP mice, respectively. They were reared in either $12 \mathrm{~h}$ light/darkness [normal reared (NR)] or constant darkness [dark reared (DR)] from postnatal day 2 (P2) or P3. All animal procedures conformed to the Guidelines on the Use of Animals in Neuroscience Research from the National Institutes of Health and the Society for Neuroscience and were approved by the Institutional Animal Care and Use Committee at the University of California, San Francisco.

Transgenic mice were identified by PCR using genomic DNA isolated from clipped tails. TrkB hypomorphic mice and Six3-Cre mice were genotyped according to Furuta et al. (2000) and Xu et al. (2000). BDNF-OE mice were genotyped using the forward primer to detect $\beta$-actin promoter: $5^{\prime}$-TCCGACCAGTGTTTGCCTTT-3'; and the reverse primer to detect human BDNF transgene: $5^{\prime}$-GGGATTGCACTTGGTCTCGT-3'. PCRs were performed with 30 cycles of $94^{\circ} \mathrm{C}$ for $30 \mathrm{~s}$, $60^{\circ} \mathrm{C}$ for $1 \mathrm{~min}$, and $72^{\circ} \mathrm{C}$ for $1 \mathrm{~min}$, followed by $72^{\circ} \mathrm{C}$ for $7 \mathrm{~min}$.

Detection and analyses of RGC dendritic structure. Retinas were harvested at three different developmental ages: P12-P13 (before eye opening, labeled as P13 for simplification), P27-P32 (2 weeks after eye open- ing, labeled as P28), and P47-P52 (5 weeks after eye opening, labeled as $\mathrm{P} 50)$. Double immunostainings were performed on whole-mounted retinas with antibodies against YFP, conjugated to Alexa Fluor fluorophore 488 (1:500; Invitrogen, San Diego, CA), and tyrosine hydroxylase (TH) (1:100; Chemicon, Temecula, CA), which labels dopaminergic neurons at the inner nuclear layer (INL) and IPL border. For each RGC, a total of 15-24 optical sections obtained with a Zeiss (Oberkochen, Germany) Pascal confocal microscope were collected at intervals of $0.8-1.5 \mu \mathrm{m}$. We followed the procedure of Badea and Nathans (2004) to quantify stratification patterns of RGC dendrites in the IPL. Measurements of the inner and outer edge of dendrites in the IPL were normalized to retinal thickness. This normalization corrected for regional differences in retinal thickness and deviations in the angle at which the whole mount was viewed. The outer edge of the IPL (IPL/INL border) was taken as the confocal $z$-section containing TH-positive processes and the inner region of TH-positive cell bodies. The inner edge of the IPL (IPL/ganglion cell layer border) was taken as the confocal $z$-section in which the RGC cell body was bisected. Off-line examination of $Z$-stacks was made of each YFP-filled RGC to identify the inner and outer edges of the RGC dendritic arbor. Previous studies support that OFF inputs are restricted to the outer $40 \%$ of the IPL in mouse retina, whereas ON inputs are restricted to the inner 60\% at P13 and in adults (Sherry et al., 2003; Ghosh et al., 2004). If the terminal dendrites of a RGC were seen exclusively in the top two-fifths of the total sections, it was classified as an OFF RGC; if the terminal dendrites of an RGC were seen in the bottom three-fifths of the total sections, it was classified as an ON RGC; RGCs that had processes in sublamina $a$ or $b$ and showed processes in more than one optical section across the sublamina $a / b$ border were classified as ON-OFF RGCs (for detailed calculations, see supplemental Fig. S1 A, available at www.jneurosci.org as supplemental material).

To trace the processes of dendritic arbors, $Z$-stack images of YFPexpressing RGCs were projected to a single two-dimensional plane using NIH Image (Abramoff et al., 2004). Based on this projected image (for an example, see Fig. $4 \mathrm{~A}$ ), dendrites were traced and the dendritic length and the number of branches were measured using ImageJ plug-in NeuroJ (Meijering et al., 2004). To validate the parameters measured in the collapsed two-dimensional images, we analyzed a group of the same RGCs using the Imaris 3D tracing program (Bitplane, Zurich, Switzerland). Our comparisons show a systematic $10-15 \%$ underestimate of total dendritic length in the two-dimensional images and no difference in the number of branches. All data sets were examined by a second observer to cross-check classification and tracing of RGCs.

Analysis of BDNF and TrkB expression in the mouse retina. Retinal sections were prepared (LaVail and Battelle, 1975; Johnson et al., 2004) and immunohistochemistry was performed as reported previously (Johnson et al., 2004). Retinal proteins were isolated, and Western blots were performed (Johnson et al., 2004). Antibodies against the following proteins were used: BDNF (1:400; Santa Cruz Biotechnology, Santa Cruz, CA) (Seki et al., 2003); protein kinase C (PKC) (1:100; Santa Cruz Biotechnology) (Johnson et al., 2004); Brn-3a (1:100; Chemicon); calretinin (1:1000; Chemicon) (Haverkamp and Wässle, 2000); SMI-32 (1: 1000; Sternberger Monoclonal, Lutherville, MD) (Nixon et al., 1989); glutamic acid decarboxylase (GAD65) (1:100; Hybridoma Bank, Iowa City, IA) (Johnson et al., 2004); calbindin (1:500; Sigma-Aldrich, St. Louis, MO); and glutamate synthetase (1:1000; BD Biosciences, San Jose, CA) (Haverkamp and Wässle, 2000). Calcium-binding protein 5 (CaBP5) (1:500) is a kind gift from Dr. Krzysztof Palczewski at Case Western Reserve University (Cleveland, OH) (Ghosh et al., 2004). TrkB antibody for Western blot is a homemade antibody by Reichardt laboratory, and TrkB antibody for immunostaining is from Chemicon (Ji et al., 2005).

Retinas were dissected for subsequent RNA isolation with Trizol reagent (Invitrogen), and the reverse transcription was performed using MessageAmp (Ambion, Austin, TX) to produce double-strand cDNA. Black Hole Quencher probe for BDNF (Biosearch Technologies, Novato, CA) was 5'-CCACAATGTTCCACCAGGTGAGAAGAGTG-3'. BDNF forward and reverse primers were as follows: $5^{\prime}$-CACTGAGTCTCCAGGACAGCAA-3' and 5' -AAATAACCATAGTAAGGAAAAGGATGGT-3'. TaqMan rodent GAPDH (Applied Biosystems, Foster City, CA) 
was used as the internal control. Real-time PCR was performed using ABI PRISM 7700 (Applied Biosystems).

TrkB full-length cDNA (clone ID, 2310034C05; RIKEN ID, ZX00131K14) was from the RIKEN FANTOM DB 2.0 microarray library (Mouse Microarray Consortium, University of California San Francisco). Digoxigenin-labeled antisense T7 and sense T3 probes (Roche, Indianapolis, IN) were prepared, and in situ hybridization was performed (Liu and Green, 2002). Peroxidase-conjugated anti-digoxigenin-peroxidase (1:500; Roche) was used to detect hybridized cRNA probes. Signals were visualized using the tyramide signal amplification system (PerkinElmer, Wellesley, MA). Immunostaining was performed after in situ hybridization.

Data analysis. Results were expressed as the mean \pm SEM. ANOVA tests were performed using GraphPad (San Diego, CA) Prism to compare multiple samples in one group, and Student's $t$ tests were performed to compare paired samples.

\section{Results}

BDNF accelerates laminar refinement of RGC dendrites

Dendritic arbors of mouse RGCs undergo progressive refinement after eye opening. Specifically, the percentage of RGCs with dendritic arbors in both sublamina $a$ and $b$ (termed ON-OFF RGCs hereafter) decreases with age after eye opening in mouse. Figure 1 confirms and extends previous studies by showing that the laminar refinement of RGC dendrites is essentially completed by P28 (Bansal et al., 2000; Tian and Copenhagen, 2003). To visualize the arborization patterns of RGC dendrites, we used a transgenic mouse line in which the expression of YFP is driven by a ganglion cell selective promoter, Thy-1 (Feng et al., 2000). The dendrites, somata, and axons of a small proportion of RGCs are filled with YFP (Feng et al., 2000; Tian and Copenhagen, 2003). Dendritic arborization patterns were identified from $Z$-stacked confocal images taken of these RGCs, after staining by YFP antibody (see Materials and Methods). Three-dimensional images of YFP-expressing RGCs (green) derived from $Z$-stack confocal images and the $90^{\circ}$ orthogonal rotations of the three-dimensional images are shown in Figure 1, $A$ and $B$, which demonstrates that the dendrites of an OFF and an ON RGC are monolaminated in the OFF and ON sublamina, respectively, whereas dendrites of an ON-OFF RGC reside in both OFF and ON sublaminas of the IPL. Because the purpose of these measurements was to assess whether each RGC could receive synapses from ON, OFF, or ON and OFF bipolar cells, we used the $40 / 60 \%$ level of the IPL to score whether processes were in the OFF or ON sublamina, a criterion valid for all of the ages we examined (Fig. 1C) (Sherry et al., 2003; Ghosh et al., 2004). Representative retinas at P13, P28, and P50 are shown in supplemental Figure S1 B (available at www. jneurosci.org as supplemental material), and the total number of RGCs studied are listed in Figure $1 D$ (also summarized in supplemental Table S1, available at www. jneurosci.org as supplemental material). At P13, just before eye opening, $51.7 \pm 2.3 \%$ of RGCs were bilaminated $\mathrm{ON}-\mathrm{OFF}$ type, whereas at $\mathrm{P} 28$, the percentage decreased significantly to $39.1 \pm 2.1 \%[p<0.01$ in one-way ANOVA post test Tukey's multiple comparison tests (TMCTs)] (Fig. 1D). Over the same period, ON RGCs increased from $39.2 \pm 2.7 \%$ at
$\mathrm{P} 13$ to $46.5 \pm 1.9 \%$ at $\mathrm{P} 28(p=0.03)$, and OFF RGCs increased from $9.1 \pm 1.4$ to $14.4 \pm 1.5 \%$ ( $p=0.03$ in Student's $t$ tests) (Fig. $1 D)$. The percentages of all three classes of RGC at P50, 5 weeks after eye opening, were not statistically different from those at P28 (Fig. 1D) ( $p>0.05$ in TMCTs), indicating that laminar refinement was essentially completed by P28.

In situ hybridization, Western blots, and immunohistochemistry analyses showed that BDNF and TrkB were expressed in the mouse inner retina, especially RGCs, during postnatal development (supplemental Fig. S2, available at www.jneurosci.org as supplemental material) (Bennett et al., 1999). This suggests that BDNF-TrkB signaling could be playing a role in RGC development well before and after eye opening. To test whether BDNF is sufficient to activate the mechanisms by which ON-OFF RGC dendrites are refined into monolaminated ON or OFF RGCs, we examined lamination patterns of dendritic arbors in mice overexpressing BDNF (BDNF-OE) (Croll et al., 1999). We first verified increases in retinal BDNF in these mice. In BDNF-OE mice, mRNA and protein levels of retinal BDNF were elevated by $81.9 \pm 25.5 \%(p=0.02)$ and $44.8 \pm 14.1 \%(p=0.02$; Student's $t$ test), respectively (Fig. $2 A$ ). Consistent with increased retinal expression assessed with Western blots, immunostainings with BDNF antibody showed that the overall level of BDNF in the inner retina was higher (Fig. $2 B$ ). Although changes in retinal BDNF mRNA is most likely of retinal origin, we cannot rule out the possibility that some BDNF protein could have been retrogradely transported in RGC axons from lateral geniculate nuclei (LGNs) or superior colliculus to RGCs in these BDNF-OE mice. Previously, Tolwani et al. (2002) showed that BDNF overexpression produced no detectable changes in TrkB expression or in TrkB signaling pathways in the brain. Similarly, we did not detect a difference in TrkB expression in retina of BDNF-OE mice (data not shown).

The overall morphology of retinas in BDNF-OE mice was very 

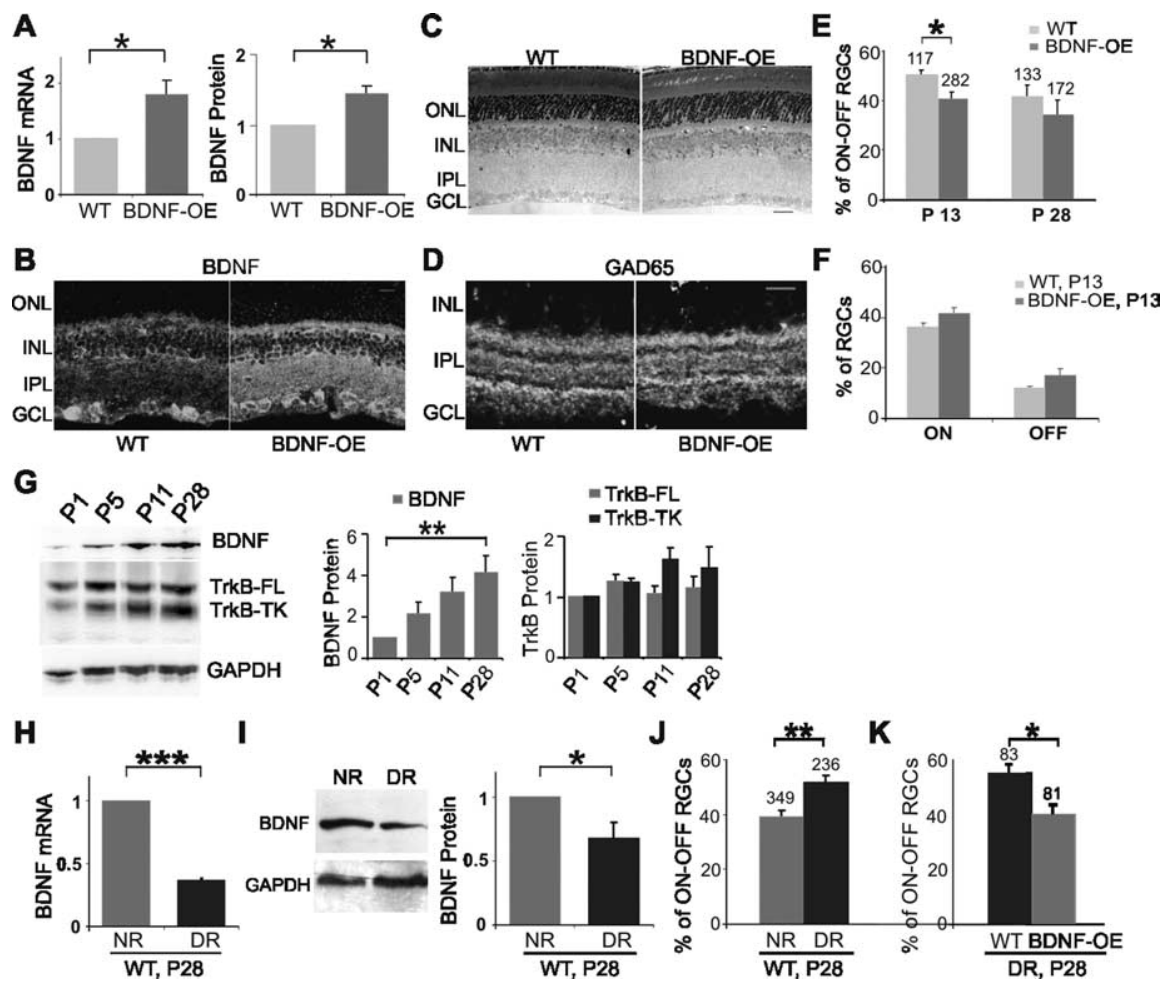

Figure 2. BDNF modulates age- and visual experience-dependent laminar refinement of RGCs. $A$, Quantification of relative retinal BDNF mRNA and protein levels in the BDNF-OE mice. RNA and protein concentrations were measured to ensure equal loading. The BDNF mRNA and protein levels were normalized to GAPDH and then calibrated to their WT controls (arbitrary unit 1; same in $\mathbf{G}-\mathbf{I}$ ). $\boldsymbol{B}$, Confocal fluorescence micrograph of BDNF immunolabeling shows that BDNF is overexpressed in BDNF-0E retina. C, Light micrograph of plastic-embedded sections of BDNF-OE and littermate control retinas. $D$, Confocal fluorescence micrograph of GAD65 immunolabeling of BDNF-OE and control retinas. Scale bar, $20 \mu \mathrm{m}$. $\boldsymbol{E}$, Comparisons of percentage of ON-OFF RGCs in BDNF-OE with their littermate control mice (WT) reared in normal light/dark cycles. The total numbers of RGCS counted at P13 and P28 are listed on the top. $\boldsymbol{F}$, Comparisons of percentages of ON and OFF RGCs in BDNF-OE with WT mice at P13. G, Quantification of relative retinal BDNF, FL TrkB, and TK TrkB levels during postnatal development $\left(N=4 ;{ }^{* *} p<0.01\right.$ in one-way ANOVA post Tukey's multiple-comparison test). $\boldsymbol{H}, \boldsymbol{I}$, Dark rearing downregulates retinal BDNF mRNA and protein levels measured by real-time $P C R(\boldsymbol{H})$ and Western blot analysis $(\boldsymbol{I})$, respectively. Representative Western blots for BDNF and the sample loading control GAPDH are shown in $\boldsymbol{I} . \boldsymbol{J}, \boldsymbol{K}$, Comparisons of percentage of ON-OFFRGCs in NR with DRWT mice $(\boldsymbol{J})$, and DRWT mice with DR BDNF-OE mice $(\boldsymbol{K}) .{ }^{*} p<0.05 ;{ }^{* *} p<0.01 ;{ }^{* * *} p<0.001$ in Student's $t$ test.

similar to that in WT mice (Fig. 2C). Markers for inner retinal neurons were not altered by BDNF overexpression, as revealed by immunostaining for anti-GAD65 (an isoform of the glutamic acid decarboxylase) (Fig. 2D), calbindin, PKC, and CaBP5 (supplemental Fig. S3A, available at www.jneurosci.org as supplemental material). No discernible changes in the outer retina were found by light microscopy (Fig. 2C). We did see effects of BDNF overexpression on $\mathrm{TH}$ amacrine cells. Consistent with previous studies of BDNF injection into rat eyes (Cellerino et al., 1998), we found that the number of $\mathrm{TH}$ amacrine cells increased in BDNF-OE mice (data not shown).

Notably, we found fewer ON-OFF RGCs in BDNF-OE mice than in their littermate controls. At P13, $41.5 \pm 2.0 \%$ of RGCs were ON-OFF in BDNF-OE mice, significantly lower than that in age-matched WT littermate controls (52.0 \pm $1.3 \%$ ) (Fig. $2 E)(p=0.02$ in Student's $t$ test). The percentages of ON RGCs and OFF RGCs together significantly increased in BDNF-OE mice $(\mathrm{ON}, 36.2 \pm 1.7$ to $41.5 \pm 2.4 \%$; and OFF, $11.8 \pm 0.8$ to $17.0 \pm 2.6 \%$ ) (Fig. 2 F). Two weeks after eye opening, at P28, $34.3 \pm 5.8 \%$ of RGCs were ON-OFF RGCs in BDNF-OE mice, not significantly different from WT littermate mice ( $41.5 \pm 4.8 \% ; p=0.38$ in Student's $t$ test) (Fig. $2 E$ ). Together, our data suggest overexpression of BDNF simply accelerates normal maturational laminar refinement. Furthermore, the effects of BDNF overexpression appear specific to RGC dendritic laminar refinement, because it did not discernibly alter retina organization, except for the upregulation of TH-positive neurons.

BDNF overexpression counteracts the effects of visual deprivation on RGC laminar refinement

The refinement of bilaminated RGC dendritic arbors to monolaminated structures is delayed by visual deprivation (Tian and Copenhagen, 2003). We further examined a possible role of BDNF in this activity-dependent remodeling of RGC arbors by (1) measuring whether retinal BDNF or TrkB levels were regulated by visual experience and (2) examining whether BDNF overexpression could prevent the retardation of dendritic arbor refinement induced by dark rearing. We first examined retinal BDNF and TrkB expression during postnatal development. Figure $2 G$ shows that BDNF expression increased significantly with age from P1 to P28 $(p<0.01$ in TMCT). In contrast, the expression of two TrkB receptor isoforms, the fulllength (FL) and truncated (TK) TrkB (Huang and Reichardt, 2001), remained constant (Fig. 2G). Similar to previous studies in rat (Pollock et al., 2001; Seki et al., 2003; Mandolesi et al., 2005), we also found that the expression level of endogenous retinal BDNF in mice is regulated by visual experience. In DR WT P28 mice, the retinal BDNF mRNA and protein levels were $36.5 \pm 6.7 \%(p<0.001)$ and $67.5 \pm 25.2 \%$ ( $p=0.04$; Student's $t$ tests $)$ of the levels in age-matched controls raised in $12 \mathrm{~h}$ dark/light cycles (NR) (Fig. $2 H, I$ ). Dark rearing did not change TrkB expression in the mouse retina (data not shown).

In dark-reared mice, more ON-OFF RGCs remained than in age-matched normally reared (NR-WT) controls at P28, confirming previous conclusions that visual experience modulates laminar refinement (Tian and Copenhagen, 2003; Landi et al., 2007). Specifically, $51.9 \pm 2.3 \%$ ON-OFF RGCs remained in the DR-WT mice versus $39.1 \pm 2.1 \%$ in NR-WT mice at P28 ( $p=0.0014$; Student's $t$ test) (Fig. $2 J)$. If visually stimulated BDNF expression played a role in normal maturational laminar refinement, overexpression of BDNF should counteract the effects of dark rearing. Consistent with this notion, in BDNF-OE mice at P28, significantly fewer RGC arbors were bilaminated compared with those in their WT littermate controls raised under identical DR conditions $(40.4 \pm 3.2$ vs $55.1 \pm 3.1 \% ; p=0.045$, Student's $t$ test) (Fig. $2 \mathrm{~K})$. Our results show that visual experience regulates retinal BDNF levels and that elevated levels of BDNF precludes the effects of visual deprivation, suggesting that visually stimulated refinement is mediated via BDNF signaling. 
Activation of BDNF through TrkB receptors regulates the conversion of bilaminated ON-OFF to monolaminated ON or OFF RGCs after eye opening

To determine whether BDNF operated through TrkB receptors and to examine refinement under conditions of diminished BDNF/TrkB signaling, we examined RGC dendritic lamination patterns in mutant mice having reduced expression of TrkB. In these TrkB mutant mice, no truncated isoforms of TrkB are expressed, whereas the expression of full-length TrkB is reduced to $25-30 \%$ in homozygous Trk$\mathrm{B}^{\mathrm{fBZ} / \mathrm{fBZ}}$ and $60-70 \%$ in heterozygous Trk$\mathrm{B}^{\mathrm{fBZ} / \mathrm{wt}}$ animals, compared with $\mathrm{TrkB}^{\mathrm{w} / \mathrm{w}}$ littermates (Xu et al., 2000). Immunostainings with TrkB and GAD65 antibodies confirmed that the expression of TrkB is reduced in the mutant retina and the structure of the inner retina is normal (Fig. $3 A, B$ ). Calbindin, CaBP5, and PKC also exhibited normal expression pattern in mice with reduced $\operatorname{TrkB}$ expression (supplemental Fig. S3B, available at www. jneurosci.org as supplemental material).

We found that the percentage of $\mathrm{ON}-$ OFF RGCs reflected the level of TrkB expression. TrkB ${ }^{\mathrm{fBZ} / \mathrm{fBZ}}$ mice had a greater percentage of ON-OFF RGCs at P28 $(67.2 \pm 8.9 \%)$ than age-matched Trk$\mathrm{B}^{\mathrm{fBZ} / \mathrm{wt}}(51.2 \pm 2.8 \% ; p=0.04$ in Student's $t$ test). In turn, $\operatorname{TrkB}{ }^{\mathrm{fBZ} / \mathrm{wt}}$ mice had a greater percentage than $\mathrm{TrkB}^{\mathrm{w} / \mathrm{w}}$ littermates $(37.4 \pm 3.8 \%)$ (Fig. $3 C$ ). The increase of ON-OFF RGCs in mice with reduced TrkB expression is mainly attributable to the decrease of ON RGCs (Fig. 3D). The percentage of ON RGCs in $\operatorname{TrkB}^{\mathrm{fBZ} / \mathrm{fBZ}}$ mice $(22.4 \pm 8.1 \%)$ is significantly lower than that in $\operatorname{TrkB}^{\mathrm{w} / \mathrm{w}}$ littermates $(49.7 \pm 3.2 \% ; p=0.04$ in Student's $t$ test $)$, whereas the percentage of OFF RGCs in $\mathrm{TrkB}^{\mathrm{fBZ} / \mathrm{fBZ}}$ mice (10.4 \pm $1.5 \%)$ was unchanged $\left(\mathrm{TrkB}^{\mathrm{w} / \mathrm{w}}, 12.9 \pm 2.0 \% ; p=0.5\right.$ in Student's $t$ test). Examining older animals, we found no significant difference in the percentage of bilaminated RGCs in animals at P50 (Fig. $3 C)(p=0.22$ in Student's $t$ test), indicating that reduced TrkB expression merely delays the process that normally refines bilaminated RGCs into monolaminated RGCs during the first 2 weeks after eye opening. Remarkably, TrkB ${ }^{\mathrm{fBZ} / \mathrm{fBZ}}$ mice at P28 had a greater percentage of ON-OFF RGCs than WT at P13 ( $67.2 \pm 8.9$ vs $51.7 \pm 2.3 \%)$, suggesting that TrkB plays a role in dendritic remodeling that occurs before eye opening. This finding supports the notion that constitutively active BDNF-TrkB signaling may regulate refinement during retinal maturation before eye opening.

To examine whether the effects we see in retina with reduced TrkB expression are a consequence of BDNF-TrkB actions within the retina and are not a result of reduced TrkB expression in RGC target tissues, such as the LGN, or did not merely reflect loss of TrkB in other tissues that could have indirect actions in retina, we deleted TrkB in mouse retina using Cre-mediated recombination. The Six3-Cre transgenic mouse used in this study contains a cre transgene under the control of the Six 3 promoter, which drives Cre recombinase expression in the developing retina and the ventral forebrain (Furuta et al., 2000). The conditional trkB knock-outs (KOs) (TrkB fff, Six3-Cre/+) were generated from crosses between mice heterozygous for the trkB floxed allele $\left(\operatorname{Tr} k B^{f /+}\right)$ and mice heterozygous for both trkB floxed allele and Six3-Cre $\left(\operatorname{TrkB}^{f /+}\right.$, Six3-Cre/+). Western blots showed that the TrkB protein was completely absent in the retina of conditional trkB-KO mice (TrkB fff, Six3-Cre/+) (Fig. 3E). The overall morphology of the retina, including the structure of the IPL, in the conditional trkB-KO mice looked normal (Fig. $3 F$, $G$; supplemental Fig. S3C, available at www.jneurosci.org as supplemental material). Interestingly, opposed to the published results of Rohrer et al. (1999), which indicated that the signal transmission in the retinal rod pathway fails in $t r k B-K O$ mice, no significant effects were observed in the electroretinogram in the conditional trkB-KO mice (R. N. Grishanin, H. Yang, X. Liu, K. DonohueRolfe, G. Nune, K. Zang, B. Xu, J. L. Duncan, M. M. LaVail, D. R. Copenhagen, and L. F. Reichardt, unpublished results). Therefore, we conclude that light-evoked signaling from photoreceptors is not compromised in the retinas of these conditional trkB-KO mice.

Thy-1-YFP transgenic mice were crossed with Six3-Cre trans- 
A

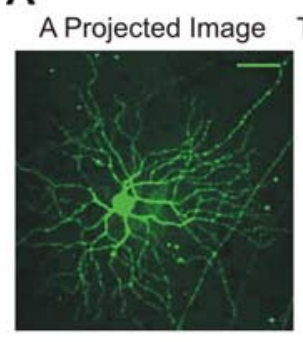

D

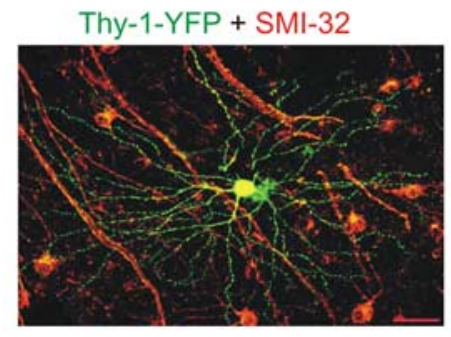

B

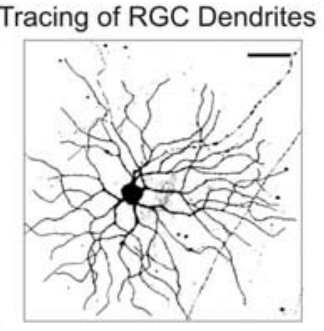

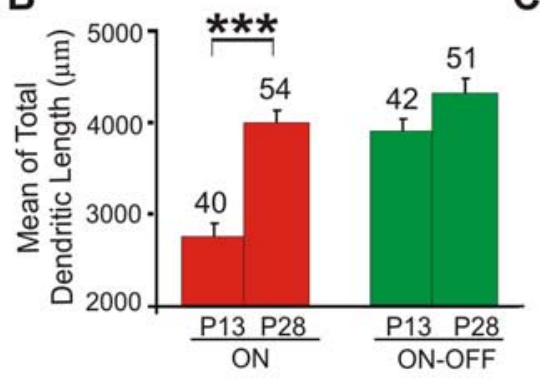

C

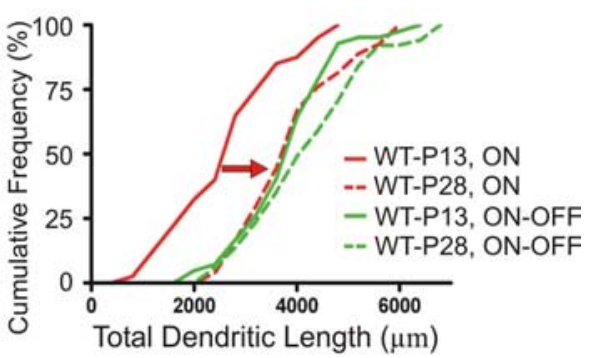

E

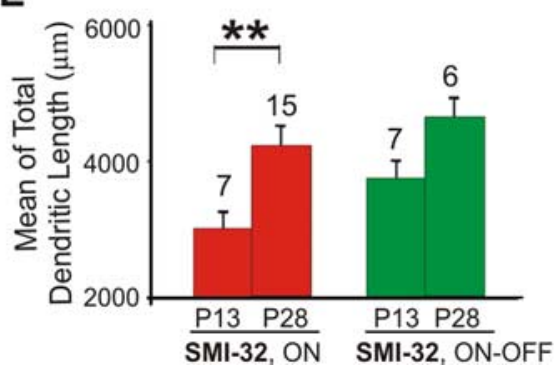

Figure 4. $0 \mathrm{~N}-\mathrm{OFF} \mathrm{RGC}$ dendritic lengths remain unchanged, whereas $\mathrm{ON} \mathrm{RGCS}$ continue to elongate after eye opening in WT retina. $A$, Left, A projected image of Z-stackimages of a representative RGC. Right, The RGC dendrites were traced with black lines. Scale bar, $50 \mu \mathrm{m}$. B, The means of total dendritic length of WT RGCs at P13 and P28. C, Relative cumulative histogram of the total dendritic length of WT RGCs. The red arrow points to the rightward shift of the distribution curves of ON RGCs from P13 to P28. D, A projected image of Z-stack images of a RGC double-immunostained with YFP (green) and SMI-32 (red) antibodies. $E$, The means of total dendritic length of SMI-32-positive RGCs. ${ }^{* *} p<0.01$; ${ }^{* * *} p<0.001$ in one-way ANOVA post Tukey's multiple-comparison tests.

genic mice and then heterozygous $\left(\operatorname{Trk} B^{f /+}\right.$, Six3-Cre/+) mice to generate the conditional trkB-KO carrying the Thy-1-YFP transgene $\left(T r k B^{f / f}\right.$, Six3-Cre/+, YFP/+). All mice were reared in normal light/dark cycles. In retinas examined at P28, we found that conditional trkB-KO mice had a greater percentage of ON-OFF RGCs at P28 (TrkB ${ }^{f / f}$, Six3-Cre/+, YFP/+; $\left.66.0 \pm 1.4 \%\right)$ than age-matched controls $\left(\mathrm{TrkB}^{\mathrm{f} /+}\right.$, Six3-Cre/+, YFP/+; $40.5 \pm$ $3.9 \% ; p<0.001$ in Student's $t$ test) (Fig. $3 H$ ). Concomitantly, the percentages of ON RGCs and OFF-RGCs were significantly decreased in the conditional trkB-KO mice: ON RGCs, $28.2 \pm 4.8$ versus $44.0 \pm 3.1 \%$; and OFF-RGCs, $5.8 \pm 3.4$ versus $15.5 \pm$ $1.7 \%(p<0.01$ in Student's $t$ test) (Fig. $3 I)$.

Because RGC laminar refinement in the $\operatorname{Trk} B^{f B Z / f B Z}$ mice was merely delayed, we could not determine whether the remaining TrkB receptors enabled refinement at a slower rate or whether an additional molecular mechanism worked in concert with BDNF and TrkB signaling. To address this issue, we examined RGC laminar refinement in the conditional trkB-KO mice at P50. Figure $3 H$ shows that $55.4 \pm 4.3 \%$ of the Thy-1-YFP-positive RGCs were bilaminated in these mice compared with $37.8 \pm 1.1 \%$ in littermate controls $\left(\mathrm{TrkB}^{\mathrm{f} /+}\right.$, Six3-Cre TrkB $^{+/+}$, Six3-Cre; and $\mathrm{TrkB}^{\mathrm{f} / \mathrm{f}}$, w/o Six3-Cre; $p=0.023$ in Student's $t$ test). That some laminar refinement appears to have occurred in P50 conditional trkB-KO mice shows BDNF and TrkB signaling does not play an exclusive role in laminar refinement. However, these results together with those showing that BDNF overexpression precludes the requirement of visual inputs to stimulate RGC laminar refinement, argues strongly for a critical role of BDNF and TrkB signaling in this process.

\section{The dendrites of ON, but not ON-OFF RGCs continue to elaborate after eye opening}

Does the refinement of ON-OFF RGCs to ON or OFF RGCs result from simple "pruning," or loss, of dendrites in the adjacent sublamina? Are more dendrites added in the appropriate sublamina? Given that exogenous BDNF modulated RGC dendritic branch numbers and complexity in Xenopus retina and rat retina explants (Bosco and Linden, 1999; Lom and Cohen-Cory, 1999), we examined RGC dendritic arbor structure quantitatively in mouse retina. To characterize the age-dependent changes of RGC dendritic structures, we traced and quantified entire dendritic arbors of individual YFP-labeled RGCs. Figure $4 A$ shows a "collapsed" image derived from projections of the $Z$-stacks of an ON-OFF RGC into one two-dimensional plane (left image). RGC dendrites in the collapsed images were traced (Fig. $4 \mathrm{~A}$, right). From these tracings, we first determined the total length of all of the dendritic branches of each RGC (725 RGCs in total). Because too few OFF RGCs were encountered to allow meaningful comparisons of these with the other cell types, we examined only ON and ON-OFF RGCs. We find that the mean total dendritic length of ON-OFF RGCs remained constant after P13. In contrast, the mean total dendritic length of ON RGCs increased 1.4 -fold between P13 and P28 ( $p<0.001$ in TMCT). Figure $4 B$ shows that the mean total dendritic length of ON-OFF RGCs was $3.9 \pm 0.1 \times 10^{3} \mu \mathrm{m}$ at $\mathrm{P} 13(N=42)$, compared with $4.3 \pm 0.2 \times$ $10^{3} \mu \mathrm{m}$ at $\mathrm{P} 28(N=51)$. The mean total dendritic length of $\mathrm{ON}$ RGCs increased from $2.8 \pm 0.2 \times 10^{3} \mu \mathrm{m}$ at P13 $(N=40)$ to $4.0 \pm 0.1 \times 10^{3} \mu \mathrm{m}$ at P28 $(N=54)$ (Fig. $\left.4 B\right)$. The cumulative distribution curve of total dendritic lengths for the ON RGCs shifted in parallel to the right (Fig. 4C), suggesting that the entire population of ON RGCs had increased dendritic lengths, and therefore, the increase was not the result of growth in only a subset of ON RGC classes.

To further rule out the possibility that the growth of ON RGC dendrites reflected a sampling bias, we examined the properties of an independently identifiable subclass of RGCs. We immunostained Thy-1-YFP retinas with SMI-32, an antibody to neurofilaments that was originally reported to selectively mark $\alpha$-type RGCs (Nixon et al., 1989) (Fig. 4D). We find that $30.9 \pm 7.4 \%$ of Thy-1-YFP-labeled neurons are labeled with SMI-32 at P28 in WT retina. Our analysis suggests that SMI-32 stains probably two more subclasses of RGC in addition to the $\alpha$-type RGCs (Coombs 
et al., 2006) (supplemental Fig. S4, available at www.jneurosci.org as supplemental material). Nonetheless, we analyzed the dendritic branching parameters in the subset of SMI-32-positive RGCs. We find that, similar to the changes of RGCs as a whole population, the mean total dendritic length increased in SMI-32-positive ON RGCs after eye opening, but remained constant in SMI-32-positive ON-OFF RGCs. Figure $4 E$ shows that the mean total dendritic length of SMI-32-positive ON RGCs increased from $3.0 \pm 0.3 \times 10^{3} \mu \mathrm{m}$ at $\mathrm{P} 13(N=7)$ to $4.2 \pm 0.3 \times 10^{3} \mu \mathrm{m}$ at P28 $(N=15)$, whereas the mean total dendritic length of SMI-32-positive ON-OFF RGCs was unchanged $\left[3.7 \pm 0.3 \times 10^{3}\right.$ $\mu \mathrm{m}$ at $\mathrm{P} 13(N=7)$, compared with $4.6 \pm$ $0.3 \times 10^{3} \mu \mathrm{m}$ at $\mathrm{P} 28(N=6) ; p>0.05$ in TMCT].

If the "pruning" of bilaminated to monolaminated arbors after eye opening is only the removal of "misplaced" dendrites, we might expect the total dendritic length of ON RGCs to remain shorter than that of ON-OFF RGCs at P28. This was not the case: our population analysis of RGC branch lengths at P28 in normally reared mice suggests that dendrites "pruned" from ON-OFF RGCs in the OFF sublamina are subsequently added to processes in the ON sublamina. Namely, the mean total dendritic length of ON-RGCs at P28 is $4.0 \pm 0.1 \times 10^{3} \mu \mathrm{m}$, which is not significantly different from $3.9 \pm 0.1 \times 10^{3}$ and $4.3 \pm 0.2 \times 10^{3} \mu \mathrm{m}$ for ON-OFF RGCs at $\mathrm{P} 13$ and $\mathrm{P} 28$, respectively (Fig. $4 B, C)(p>0.05$ in TMCT); and the mean total dendritic length of SMI-32-positive ON RGCs is $4.2 \pm 0.3 \times 10^{3} \mu \mathrm{m}$, which is also not significantly different from $4.6 \pm 0.3 \times 10^{3}$ for SMI-32-positive ON-OFF RGCs at P28 (Fig. $4 E)$ ( $p>0.05$ in TMCT). Therefore, new dendritic processes are likely to be added to balance those that are lost. These findings are consistent with the idea that the dendritic maturation in monolaminated ON RGCs reflects in part a loss of dendritic branches from formerly bilaminated RGC arbors in sublamina $a$ as well as an increase of dendrites in sublamina $b$ (see Fig. 7, diagram).

The growth of ON RGC dendrites is mainly attributable to the increase of branch numbers after eye opening

An overall increase of dendritic length can be divided into two related but independent processes: increased branch number and branch elongation. Our analysis indicates that the developmental increase of total dendritic length of ON RGCs mainly results from increased branch numbers and not elongation of individual branches. The mean total branch number in the dendritic arbors of ON RGCs increased from $73.3 \pm 4.9$ at P13 to $91.4 \pm 4.9$ at $\mathrm{P} 28$ (Fig. $5 A)(p<0.001$ in TMCT). The parallel shift of the cumulative distribution curve of total branches for the ON RGCs (Fig. $5 B$ ) suggests that the increase was not the result of growth in a subset of ON RGC classes. In contrast, the branch numbers of ON-OFF RGCs were not altered significantly during this period (91.1 \pm 5.4 at P13 vs $101.9 \pm 4.2$ at P28) (Fig. 5A). The mean branch lengths of $\mathrm{ON}$ and $\mathrm{ON}-\mathrm{OFF}$ RGCs did not change significantly between P13 and P28 (ON, $40.4 \pm 2.1$ vs $46.8 \pm 2.1 \mu \mathrm{m}$; ON-OFF, $47.2 \pm 2.5$ vs $44.6 \pm 2.5 \mu \mathrm{m}$ ) (Fig. $5 D, E$ ).

SMI-32-positive ON RGCs and ON-OFF RGCs exhibited similar growth patterns to the whole population of Thy-1-YFP-
B
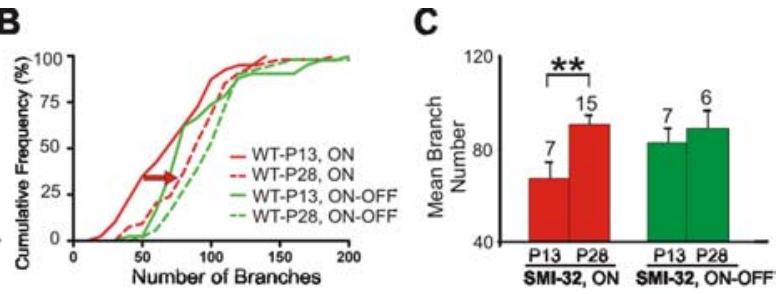

$\mathbf{F}$

E
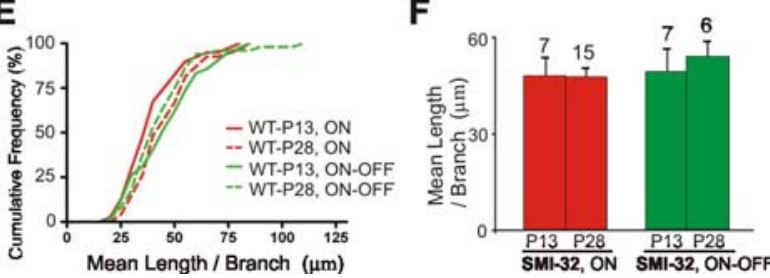

Figure 5. The number of branches increases, whereas the mean length per branch of ON RGCs remains unchanged after eye opening. $A, D$, Comparisons of the means branch numbers $(\boldsymbol{A})$ and mean length/branch $(\boldsymbol{D})$ of $0 \mathrm{~N}$ and $0 \mathrm{~N}-0 \mathrm{FF} \mathrm{RGCS}$ at P13 and B, $\boldsymbol{E}$, Relative cumulative histograms of the branch numbers and mean length/branch of ON and ON-OFF RGCS at P13 and P28. $\boldsymbol{C}, \boldsymbol{F}$, Comparisons of the means branch numbers $(\boldsymbol{C})$ and mean length/branch $(\boldsymbol{F})$ of SMI-32-positive ON and ON-OFF RGCS at 0.01 in one-way ANOVA post Tukey's multiple-comparison tests.

labeled RGCs (Fig. 5C,F). The mean total branch number in the dendritic arbors of SMI-32-positive ON RGCs increased from $67.1 \pm 7.2$ at P13 to $90.4 \pm 4.3$ at P28 (Fig. $5 C)(p<0.05$ in TMCT), and the mean total branch number of SMI-32-positive ON-OFF RGCs was not altered during this period (82.4 \pm 6.6 at $\mathrm{P} 13$ vs $88.8 \pm 7.9$ at $\mathrm{P} 28 ; p>0.05$ in TMCT) (Fig. $5 C$ ). The mean branch lengths of SMI-32-positive ON and ON-OFF RGCs did not change significantly between $\mathrm{P} 13$ and $\mathrm{P} 28(\mathrm{ON}, 47.7 \pm 5.7$ vs $47.3 \pm 3.2 \mu \mathrm{m}$; ON-OFF, $48.8 \pm 7.6$ vs $53.7 \pm 5.0 \mu \mathrm{m} ; p>0.05$ in TMCTs) (Fig. 5F).

\section{BDNF and TrkB modulate experience-dependent RGC dendritic branching}

Because ON RGCs continue to grow by adding more branches after eye opening, we first determined whether visual deprivation affects this age-dependent increase in branch number of $\mathrm{ON}$ RGC dendritic arbors. We find that ON RGCs of dark-reared animals have fewer dendritic branches. The number of ON RGC dendritic branches in DR mice $(80.0 \pm 4.4 ; N=34)$ at P28 was significantly lower than in NR mice (94.6 $\pm 4.7 ; N=42)$ (Fig. $6 \mathrm{~A}$ ). Dark rearing did not change the mean branch number of ON-OFF RGCs [96.2 \pm 4.8 in DR $(N=38)$ vs $101.1 \pm 3.8$ in NR $(N=43)$ ] (Fig. 6A). SMI-32-positive RGCs also exhibit similar changes as the whole population of RGCs. The branch numbers of SMI-32-positive ON RGCs significantly decreased in DR WT mice (NR, 90.4 $\pm 4.3, N=15 ; \mathrm{DR}, 66.1 \pm 8.7, N=8$ ), whereas ON-OFF RGCs remain unchanged $(\mathrm{NR}, 88.8 \pm 7.9, N=6$; DR, $94.4 \pm 12.1, N=7$ ) (Fig. $6 B$ ).

We examined whether BDNF and TrkB signaling affects RGC dendritic branching. Overexpression of BDNF increases dendritic branching of ON RGCs during postnatal development. The mean number of dendritic branches of ON RGCs in BDNF-OE mice at $\mathrm{P} 13(88.3 \pm 4.4 ; N=25)$ was significantly higher than in the WT controls (73.2 $\pm 4.9 ; N=40 ; p>0.05$ in Student's $t$ test). In contrast, branch numbers in ON-OFF RGCs were not significantly different (BDNF-OE, $106.8 \pm 5.9, N=21$; WT, $91.1 \pm$ 5.4, $N=42 ; p>0.05$ in Student's $t$ test) (Fig. $6 C$ ). SMI-32positive ON RGCs also exhibited a significant increase of branch numbers in BDNF-OE mice, whereas ON-OFF RGCs remain unchanged (data not shown).

Reduced TrkB expression suppressed the maturational in- 
A

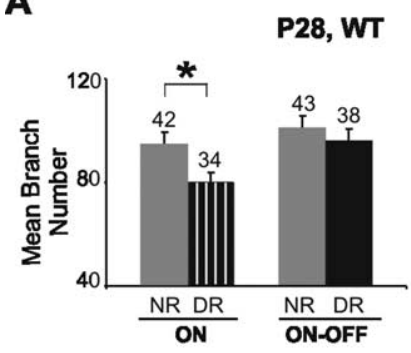

B

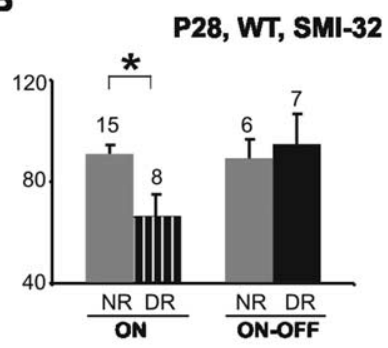

C

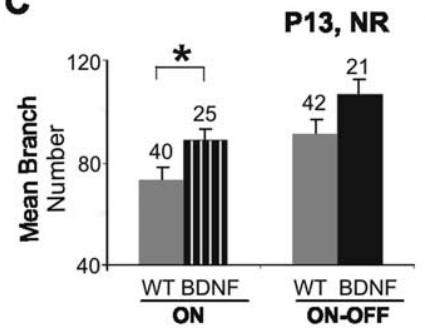

D

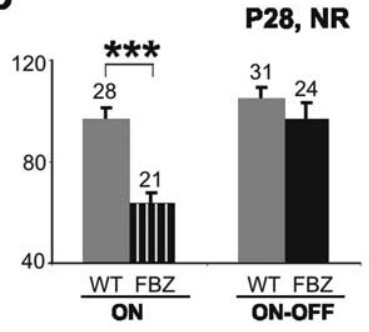

$\mathbf{F}$

E

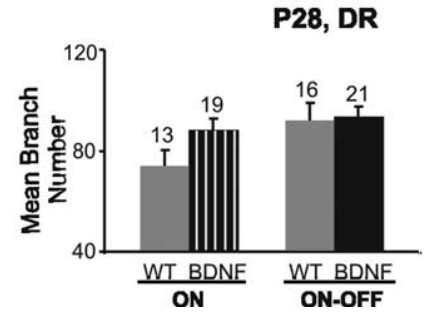

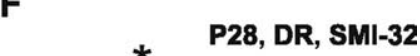

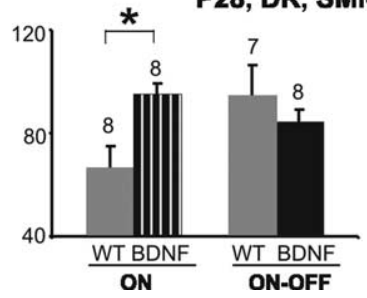

Figure 6. BDNF and TrkB modulate experience-dependent dendritic branch elongation of ON RGCS. $A, B$, Dark rearing decreases the branch numbers of ON RGCs and SMI-32-positive ON RGCs at P28. C, Overexpression of BDNF increases the branch numbers of ON-RGC at P13. D, Reduced TrkB expression results in decreased branch numbers in ON RGCs at P28. $E$, Mean branch numbers of ON RGCS in DR BDNF-OE mice are not significantly different from DR WT controls. $\boldsymbol{F}$, Mean branch numbers of SMI-32-positive ON RGCS in DR BDNF-OE mice are higher than their DR WT controls. The branch numbers of ON-OFF RGCs are comparable in above conditions. ${ }^{*} p<0.05 ;{ }^{* *} p<0.01 ;{ }^{* * *} p<0.001$ in Student's $t$ test.

crease of branch numbers in ON RGCs. We examined RGC branch numbers in mice with reduced TrkB expression reared in normal light/dark cycles at $\mathrm{P} 28$. We find that $\mathrm{P} 28$-aged mice with reduced TrkB expression have fewer numbers of ON RGC dendritic branches: $62.8 \pm 4.4$ in $\operatorname{TrkB}^{\mathrm{fBZ} / \mathrm{fBZ}}(N=21)$ compared with $96.7 \pm 4.5$ in $\mathrm{TrkB}^{\mathrm{w} / \mathrm{w}}$ at $\mathrm{P} 28(N=28)$ (Fig. $\left.6 D\right)$. Branch numbers of ON-OFF RGCs were not significantly different in mice with reduced TrkB expression $\left[96.8 \pm 6.6\right.$ in $\operatorname{TrkB}^{\mathrm{fBZ} / \mathrm{fBZ}}$ $(N=24)$ vs $104.7 \pm 4.7$ in $\left.\operatorname{TrkB}^{\mathrm{w} / \mathrm{w}}(N=31)\right]$ (Fig. $\left.6 D\right)$.

To test whether overexpression of BDNF could preclude the requirement for visual inputs to stimulate RGC dendritic branching, we examined the RGC dendritic branching in DR BDNF-OE mice at P28. The total branch numbers of SMI-32-positive ON RGCs were significantly increased in BDNF-OE mice [BDNF, $94.6 \pm 4.6(N=8)$; vs WT, $66.1 \pm 8.7(N=8) ; p=0.015$ in Student's $t$ test] (Fig. $6 F$ ), suggesting that the dendritic branching of SMI-32-positive ON RGCs is regulated by BDNF. Surprisingly, the mean total branch number of all Thy-1-YFP-positive ON RGCs of DR BDNF-OE mice is not significantly different from DR WT controls [BDNF-OE, $88.1 \pm 4.5(N=19)$; vs WT, $79.9 \pm 4.5(N=13) ; p=0.08$ in Student's $t$ test] (Fig. 6E). A greater sample size of DR BDNF-OE mice would need to be studied to determine whether the ability of BDNF overexpression to

override dark rearing is only specific to SMI-32-positive RGCs or holds true for all ON RGCs.

\section{Discussion}

Here, we present experimental evidence that BDNF and TrkB signaling modulates developmental and visual experiencedependent laminar refinement and branching of RGC dendrites (Fig. 7, model). Reduced TrkB or deleted expression, respectively, delays or suppresses the laminar refinement of bilaminated RGC dendrites in mice with normal visual experience. Overexpression of BDNF accelerates laminar remodeling and, importantly, precludes the retardation of laminar refinement in darkreared animals. Because a population of ON-OFF RGCs remain after laminar refinement, this suggests that there could be two distinguishable classes on $\mathrm{ON}-\mathrm{OFF}$ cell, one that is responsive to dark rearing and/or BDNF/TrkB activation and another that is not. Furthermore, after eye opening in normally reared mice, the dendrites of ON RGCs, but not ON-OFF RGCs, elongate by the addition of branches. Visual deprivation or reduced TrkB expression decreases and high BDNF expression increases branch formation in the monolaminated ON RGCs. Because visual experience and BDNF activation of TrkB affects laminar refinement of ON-OFF RGCs but branch numbers of only ON RGCs, we suggest that activity and neurotrophins regulate different aspects of dendritic remodeling in cell class-specific ways. The two simplest possibilities are that one class of ON-OFF cells is sensitive to $\mathrm{BDNF} / \mathrm{TrkB}$ activation and these cells lose dendrites in sublamina $a$ and simultaneously add branches in sublamina $b$. Alternatively, one class of ON-OFF RGCs simply lose their dendritic processes in sublamina $a$ and a separate class of ON cells are stimulated to extend branch numbers by visual experience or $\mathrm{BDNF} / \mathrm{TrkB}$ activation.

\section{Thy-1-YFP mice as a model to study RGC maturation and refinement}

Morphologically, 11-14 subtypes of mouse RGCs have been reported (Masland, 2001; Sun et al., 2002; Badea and Nathans, 2004; Coombs et al., 2006). We found that Thy-1-YFP transgenelabeled RGCs were members of most of the RGC subtypes reported previously, except that a subpopulation of large RGCs having diameters of dendritic coverage from 350 to $450 \mu \mathrm{m}$ (Sun et al., 2002) was not represented in our YFP-labeled RGC samples (data not shown).

Comparisons of our findings with other studies of mouse retina that used alternative marking techniques yield approximately comparable percentages of ON-OFF RGCs in WT animals. Using the "DiOlistic" method, $\sim 25 \%$ lipophilic dye (DiI)-labeled RGCs were classified as bistratified, ON-OFF RGCs (Sun et al., 2002). This study by Sun et al. (2002) only provided data on the center point of arbor stratification within the IPL, which would underestimate the number of RGCs having dendritic arbors positioned across the sublamina $a / b$ border. Using pharmacologically controlled, Cre-mediated DNA recombination to generate alkaline phosphatase-marked cells, Badea and Nathans (2004) estimated that $\sim 50 \%$ of RGCs would be classified as ON-OFF RGCs. Our anatomical approach using Thy-1-YFP transgenic mice yields similar percentages of ON-OFF RGCs $(\sim 40 \%)$ (Figs. 1-3). Using another strain of Thyl-YFP mice, Landi et al. (2007) report $\sim 31 \%$ ON-OFF RGCS at P30. The reasonable agreement produced by the three different techniques indicates that the Thy-1-YFP transgenic line provides representative samples of RGCs to allow analysis of the laminar morphology of these retinal neurons in vivo. 
We checked that the experimental differences we observed between groups of animals were not accounted for by the regulation of Thy-1-YFP expression. First, Western blots indicated that dark rearing did not alter Thy-1-YFP expression (data not shown). Second, we found that the percentages of RGCs double labeled by SMI-32 and YFP antibodies were not altered by dark rearing or BDNF overexpression. For example, $25.7 \pm 1.7 \%$ of Thy-1YFP RGCs were double labeled with SMI-32 in BDNF-OE mice, compared with $30.9 \pm 7.4 \%$ of their WT controls ( $p=0.6$ in Student's $t$ test). Third, cell death does not appear to affect the expression of Thy-1-YFP in RGCs. RGC death peaks at approximately P2-P6 (Young, 1984; Linden and Pinto, 1985), and BDNF and TrkB signaling does not affect the final number of RGCs (Pollock et al., 2003). We found comparable numbers of YFPexpressing RGCs in dark-reared, BDNFoverexpressing, TrkB-hypomorphic, and conditional trkB-KO mice at P13, P28, and P50. We interpret these findings as evidence that the expression pattern of YFP driven by the Thy-1 promoter remained stable under different genetic backgrounds and rearing conditions.

\section{How could visual-evoked activity and BDNF signaling regulate RGC morphology?}

Light-evoked modulation of excitatory glutamatergic bipolar cells is an obvious mechanism to organize RGC dendritic arbors. However, no consensus has emerged as to whether or how afferent inputs from bipolar cells play a role in organizing RGC dendritic arbors. Our results demonstrate that the morphology of $\mathrm{ON}$ and OFF bipolar cells is not discernibly altered by the visual experience (data not shown), nor by BDNF and TrkB signaling (Figs. 3, 4; supplemental Fig. S3, available at www.jneurosci.org as supplemental material). Some previous pharmacological studies argue that glutamatergic visual signals from $\mathrm{ON}$ bipolar cells may be required for RGC dendritic pruning. Chronic intraocular injections of APB (DL-2-amino-4-phosphonobutyric acid), an agonist for the metabotropic glutamate receptor 6 (mGluR6) of ON bipolar cells and for other class III metabotropic receptors in the retina, delays the emergence of $\mathrm{ON}-\mathrm{OFF}$ stratification in a reversible manner (Bodnarenko and Chalupa, 1993; Bodnarenko et al., 1995). However, knock-out of the mGluR6 in ON bipolar cells has minimal effects on RGC lamination refinement (Tagawa et al., 1999), providing evidence against bipolar synaptic transmission being necessary for RGC laminar refinement.

It is also unclear whether different types of amacrine cells could affect the maturation of RGC dendritic arbors. During early development, RGCs first establish synapses with amacrine cells, and later with bipolar cells (Sernagor et al., 2001). Before the retina becomes light sensitive, the starburst amacrine network mediates spontaneous retinal waves through the excitatory neurotransmitter, acetylcholine (Feller et al., 1996; Zheng et al., 2004). In mice lacking nicotinic acetylcholine receptor (nAChR) subunits $\alpha 3$ and $\beta 2$, the gross segregation of RGC dendrites into ON and OFF sublaminas were normal, but the refinement of individual RGC dendrites is delayed, suggesting that retinal waves mediated by $\mathrm{nAChRs}$ play a limited role in the laminar refinement of RGC dendrites (Bansal et al., 2000).
Our results also showed that BDNF and TrkB signaling modulates maturation of $\mathrm{TH}$-immunoreactive amacrine cells (data not shown); however, there is no direct evidence that dopamine affects RGC laminar refinement and maturation (for review, see Witkovsky, 2004).

Could BDNF and TrkB signaling control RGC dendritic remodeling by altering light-evoked signaling from photoreceptors or spiking activity of RGCs? Rohrer et al. (1999) reported that synaptic signaling from photoreceptors to bipolar cells was compromised in TrkB knock-out mice (Rohrer et al., 1999). Contrary to this report, we find that photoreceptor to bipolar signaling is normal in the retina-specific conditional trkB knock-out mice (Grishanin, Yang, Liu, Donohue-Rolfe, Nune, Zang, Xu, Duncan, LaVail, Copenhagen, and Reichardt, unpublished results). Therefore, the delay in laminar refinement and branch formation we observe here is not attributable to a loss of visual signaling through photoreceptor-bipolar cell synapses. It is also known that BDNF activation of TrkB can enhance spiking activity of neurons (Huang and Reichardt, 2001); however, Du and Poo (2004) showed direct application of BDNF into retina had no effect on RGC activity in Xenopus. Furthermore, neither the spontaneous discharge rates nor amplitudes of light-evoked responses of RGCs was changed by BDNF levels in rodent retina (Mandolesi et al., 2005). Our preliminary experiments in which multielectrode array recordings were used to examine and compare spontaneous spiking of RGCs in BDNF-OE mice corroborate these findings (WT, 1.77 spikes/min; vs BDNF-OE, 1.93 spikes/min; $p=0.78$ in Student's $t$ test). Thus, a model in which BDNF and TrkB signaling merely regulates overall activity and responsiveness of retinal circuitry does not appear to explain the remodeling we report in this study. Instead, visual activity may modify constitutively active BDNF levels to retard or accelerate RGC laminar refinement.

\section{Age- and experience-dependent maturation of different} subtype RGCs

Different subtypes of RGCs mature at different rate during the postnatal development in the mammalian retinas (Sernagor et 
al., 2001). In kitten, the arbors of $\gamma$ RGCs are adult-like at birth, whereas the dendritic fields of $\alpha$-cells reach their adult dimensions 3 weeks after birth, around which time $\beta$-cell dendritic expansion begins (Dann et al., 1988; Ault and Leventhal, 1994). The dendritic fields and the total dendritic length of small field and medium field RGCs increases faster than those of $\alpha$ RGCs in corresponding retinal positions in rabbit retina (Wong, 1990). In mouse, most subtypes of RGCs are present before eye opening (Diao et al., 2004), but the temporal and spatial changes of RGC dendritic morphology after eye opening has not been characterized in detail. Here, we demonstrate that different types of RGCs in mouse undergo differential remodeling during postnatal maturation. The underlying molecular and cellular mechanisms are mostly unknown. Because ON RGCs can be identified soon after birth and a percentage of ON-OFF RGCs remains in adult, it is likely that subclasses of each type of RGCs are determined by cell-autonomous genetic programs (Sernagor et al., 2001). DNA microarrays to profile genes in different subtypes of RGCs may help to identify genes required for RGC subtype differentiation and maturation both morphologically and functionally.

Our detailed analysis of RGC dendritic structures reveals that the branch formation of monolaminated ON RGCs is regulated by visual experience. We showed that BDNF and TrkB regulate dendritic branching of ON RGCs. Dark rearing blocked the increase of branch numbers, and BDNF overexpression overrides the dark-rearing effects on SMI-32-positive ON RGCs. Because BDNF and TrkB signaling and dark rearing affect branch numbers of ON RGCs and not ON-OFF RGCs, this demonstrates that subclasses of RGCs are more plastic or more sensitive to neurotrophin regulation. These results and previous studies suggest that the growth of RGC dendritic trees depends on the RGC subtype. A parsimonious model would suggest that a subclass of ON-OFF RGCs that can become ON RGCs are sensitive to BDNF and TrkB signaling.

\section{Potential molecular signaling mechanisms underlying RGC dendritic maturation}

BDNF levels are known to be regulated in an activity-dependent manner in the nervous system ( $\mathrm{Lu}, 2003)$. In the visual system, maturation controls transcription of BDNF; mRNA levels of BDNF increase in retina and visual cortex after eye opening (Castren et al., 1992; Seki et al., 2003). Dark rearing decreases mRNA levels of BDNF in both of these areas (Castren et al., 1992; Seki et al., 2003; this study). BDNF protein levels are reduced in the retinas of dark-reared animals or in retinas of lid-sutured eyes (Seki et al., 2003; Mandolesi et al., 2005; this study). TrkB protein expression in retina and visual cortex is not altered by dark rearing (Viegi et al., 2002). These previous findings together with our results support the idea that visual stimulation during postnatal development regulates the transcription and protein levels of $\mathrm{BDNF}$ in retina, and BDNF, acting at TrkB receptors, is an important signaling molecule modulating visual experiencedependent plasticity of RGCs. A recent study by Landi et al. (2007) supports the notion that BDNF-TrkB signaling can be stimulated not only by visual inputs but also by raising mothers and pups in an enriched physical environment. Despite good evidence for activity-dependent regulation of BDNF levels, it is probable that there is a developmentally regulated expression of BDNF that is independent of activity. As evidence, the higher percentage of ON-OFF RGCs in P28 TrkB conditional knockout mice than in P13 WT mice ( 66 vs 52\%) supports the idea that BDNF-TrkB signaling may be activating laminar refinement well before eye opening and the onset visual responsiveness of the retina.

One unresolved question in our findings is whether BDNF/ TrkB signaling is the only neurotrophic pathway underlying the laminar refinement and branching of RGC arbors. It has been shown that pro-BDNF can activate $\mathrm{p} 75^{\mathrm{NTR}}$ in hippocampus (Woo et al., 2005), and p75 NTR negatively modulates dendritic morphology of hippocampal pyramidal neurons in adult (Zagrebelsky et al., 2005). Our results argue that p75NTR signaling is likely of secondary importance. Also, we cannot rule out that neurotrophins other than BDNF may play a role in maturational or activity-dependent refinement of RGC arbors. For example, neurotrophin-3 can also regulate dendritic growth of different pyramidal cell dendrites in organotypic brain slices (McAllister et al., 1997). However, with conditional knock-out of trkB gene in the retina, $66 \%$ of total RGCs remained as ON-OFF RGCs, which is much higher than $52 \%$ ON-OFF RGCs in darkreared animals, and higher than $51.7 \%$ ON-OFF RGCs at P13. Therefore, RGC refinement is blocked by blocking TrkB pathways. It should be noted here that only $\sim 70 \%$ of the RGCs at birth are bilaminated and thus potential candidates for laminar refinement. An analysis of RGC dendritic arbors in P0 mice shows that, at birth, $31 \%$ of the RGCs are already monolaminated (Diao et al. 2004). Given the actions produced by the upregulation and downregulation of BDNF/TrkB signaling achieved by using mice with BDNF overexpression, and reduced TrkB expression, and deletion of TrkB in the retina, it is nonetheless very likely that BDNF and TrkB signaling plays a critical role in refinement of RGCs. Given the actions of visual deprivation on BDNF levels and remodeling of RGC arbors, we interpret these findings as evidence that light stimulation regulates BDNF expression, which, in turn, induces laminar refinement and increased numbers of dendritic branches. However, we cannot rule out that light is acting via a pathway independent of BDNF.

\section{Do BDNF/TrkB-mediated retinal changes affect plasticity observed in the cortex?}

Our results raise an interesting question of whether activity and BDNF signaling via TrkB receptors in retina play a role in regulating the plasticity of higher visual centers. In mouse, development of precise maps in visual cortex was shown to require patterned spontaneous activity in retina (Cang et al., 2005). Also, blockade of spontaneous retinal activity by tetrodotoxin around the time of eye opening, or visual deprivation after 1 week of vision, disrupted synaptic strengthening and pruning of excess retinal afferents in LGN (Hooks and Chen, 2006). These above studies indicate that early spontaneous activity in the eye or visual experience could alter the synaptic connections in higher visual centers, yet little is known of the underlying molecular mechanism (Cang et al., 2005; Hooks and Chen, 2006). Interestingly, exogenous BDNF protein injected into a visually deprived eye, or reduced endogenous BDNF expression in the nondeprived eye, was found to counteract the ocular dominance shift induced by eyelid closure (Mandolesi et al., 2005). Mandolesi et al. (2005) speculated that an unidentified change in retinal function could regulate cortical plasticity. Because we document BDNF and TrkB signaling induced changes in RGC dendritic arbor morphology, our findings add credence that BDNF- and TrkBmediated changes in retinal morphology and function might underlie at least part of the plastic changes observed in cortex.

\section{References}

Abramoff MD, Magelhaes PJ, Ram SJ (2004) Image processing with ImageJ. Biophotonics Int 11:36-42. 
Amthor FR, Oyster CW, Takahashi ES (1984) Morphology of on-off direction-selective ganglion cells in the rabbit retina. Brain Res 298:187-190.

Ault SJ, Leventhal AG (1994) Postnatal development of different classes of cat retinal ganglion cells. J Comp Neurol 339:106-116.

Badea TC, Nathans J (2004) Quantitative analysis of neuronal morphologies in the mouse retina visualized by using a genetically directed reporter. J Comp Neurol 480:331-351.

Bansal A, Singer JH, Hwang BJ, Xu W, Beaudet A, Feller MB (2000) Mice lacking specific nicotinic acetylcholine receptor subunits exhibit dramatically altered spontaneous activity patterns and reveal a limited role for retinal waves in forming ON and OFF circuits in the inner retina. J Neurosci 20:7672-7681.

Bennett JL, Zeiler SR, Jones KR (1999) Patterned expression of BDNF and NT-3 in the retina and anterior segment of the developing mammalian eye. Invest Ophthalmol Vis Sci 40:2996-3005.

Bodnarenko SR, Chalupa LM (1993) Stratification of ON and OFF ganglion cell dendrites depends on glutamate-mediated afferent activity in the developing retina. Nature 364:144-146.

Bodnarenko SR, Jeyarasasingam G, Chalupa LM (1995) Development and regulation of dendritic stratification in retinal ganglion cells by glutamate-mediated afferent activity. J Neurosci 15:7037-7045.

Bodnarenko SR, Yeung G, Thomas L, McCarthy M (1999) The development of retinal ganglion cell dendritic stratification in ferrets. NeuroReport 10:2955-2959.

Bosco A, Linden R (1999) BDNF and NT-4 differentially modulate neurite outgrowth in developing retinal ganglion cells. J Neurosci Res 57:759-769.

Cang J, Renteria RC, Kaneko M, Liu X, Copenhagen DR, Stryker MP (2005) Development of precise maps in visual cortex requires patterned spontaneous activity in the retina. Neuron 48:797-809.

Castren E, Zafra F, Thoenen H, Lindholm D (1992) Light regulates expression of brain-derived neurotrophic factor mRNA in rat visual cortex. Proc Natl Acad Sci USA 89:9444-9448.

Cellerino A, Pinzon-Duarte G, Carroll P, Kohler K (1998) Brain-derived neurotrophic factor modulates the development of the dopaminergic network in the rodent retina. J Neurosci 18:3351-3362.

Coombs J, van der List D, Wang GY, Chalupa LM (2006) Morphological properties of mouse retinal ganglion cells. Neuroscience 140:123-136.

Croll SD, Suri C, Compton DL, Simmons MV, Yancopoulos GD, Lindsay RM, Wiegand SJ, Rudge JS, Scharfman HE (1999) Brain-derived neurotrophic factor transgenic mice exhibit passive avoidance deficits, increased seizure severity and in vitro hyperexcitability in the hippocampus and entorhinal cortex. Neuroscience 93:1491-1506.

Dann JF, Buhl EH, Peichl L (1988) Postnatal dendritic maturation of $\alpha$ and $\beta$ ganglion cells in cat retina. J Neurosci 8:1485-1499.

Diao L, Sun W, Deng Q, He S (2004) Development of the mouse retina: emerging morphological diversity of the ganglion cells. J Neurobiol 61:236-249.

Du JL, Poo MM (2004) Rapid BDNF-induced retrograde synaptic modification in a developing retinotectal system. Nature 429:878-883.

Famiglietti Jr EV, Kolb H (1976) Structural basis for ON-and OFF-center responses in retinal ganglion cells. Science 194:193-195.

Feller MB, Wellis DP, Stellwagen D, Werblin FS, Shatz CJ (1996) Requirement for cholinergic synaptic transmission in the propagation of spontaneous retinal waves. Science 272:1182-1187.

Feng G, Mellor RH, Bernstein M, Keller-Peck C, Nguyen QT, Wallace M, Nerbonne JM, Lichtman JW, Sanes JR (2000) Imaging neuronal subsets in transgenic mice expressing multiple spectral variants of GFP. Neuron 28:41-51.

Furuta Y, Lagutin O, Hogan BL, Oliver GC (2000) Retina- and ventral forebrain-specific Cre recombinase activity in transgenic mice. Genesis 26:130-132.

Ghosh KK, Bujan S, Haverkamp S, Feigenspan A, Wässle H (2004) Types of bipolar cells in the mouse retina. J Comp Neurol 469:70-82.

Haverkamp S, Wässle H (2000) Immunocytochemical analysis of the mouse retina. J Comp Neurol 424:1-23.

Hooks BM, Chen C (2006) Distinct roles for spontaneous and visual activity in remodeling of the retinogeniculate synapse. Neuron 52:281-291.

Horch HW, Katz LC (2002) BDNF release from single cells elicits local dendritic growth in nearby neurons. Nat Neurosci 5:1177-1184.
Horch HW, Kruttgen A, Portbury SD, Katz LC (1999) Destabilization of cortical dendrites and spines by BDNF. Neuron 23:353-364.

Huang EJ, Reichardt LF (2001) Neurotrophins: roles in neuronal development and function. Annu Rev Neurosci 24:677-736.

Ji Y, Pang PT, Feng L, Lu B (2005) Cyclic AMP controls BDNF-induced TrkB phosphorylation and dendritic spine formation in mature hippocampal neurons. Nat Neurosci 8:164-172.

Johnson J, Sherry DM, Liu X, Fremeau Jr RT, Seal RP, Edwards RH, Copenhagen DR (2004) Vesicular glutamate transporter 3 expression identifies glutamatergic amacrine cells in the rodent retina. J Comp Neurol 477:386-398.

Landi S, Cenni MC, Maffei L, Berardi N (2007) Environmental enrichment effects on development of retinal ganglion cell dendritic stratification require retinal BDNF. PLoS ONE 2:e346.

LaVail MM, Battelle BA (1975) Influence of eye pigmentation and light deprivation on inherited retinal dystrophy in the rat. Exp Eye Res 21:167-192.

Linden R, Pinto LH (1985) Developmental genetics of the retina: evidence that the pearl mutation in the mouse affects the time course of natural cell death in the ganglion cell layer. Exp Brain Res 60:79-86.

Liu X, Green CB (2002) Circadian regulation of nocturnin transcription by phosphorylated CREB in Xenopus retinal photoreceptor cells. Mol Cell Biol 22:7501-7511.

Lom B, Cohen-Cory S (1999) Brain-derived neurotrophic factor differentially regulates retinal ganglion cell dendritic and axonal arborization in vivo. J Neurosci 19:9928-9938.

Lu B (2003) BDNF and activity-dependent synaptic modulation. Learn Mem 10:86-98.

Mandolesi G, Menna E, Harauzov A, von Bartheld CS, Caleo M, Maffei L (2005) A role for retinal brain-derived neurotrophic factor in ocular dominance plasticity. Curr Biol 15:2119-2124.

Masland RH (2001) Neuronal diversity in the retina. Curr Opin Neurobiol 11:431-436.

Maslim J, Stone J (1988) Time course of stratification of the dendritic fields of ganglion cells in the retina of the cat. Brain Res Dev Brain Res 44:87-93.

McAllister AK, Katz LC, Lo DC (1997) Opposing roles for endogenous BDNF and NT-3 in regulating cortical dendritic growth. Neuron 18:767-778.

McAllister AK, Katz LC, Lo DC (1999) Neurotrophins and synaptic plasticity. Annu Rev Neurosci 22:295-318.

Meijering E, Jacob M, Sarria JC, Steiner P, Hirling H, Unser M (2004) Design and validation of a tool for neurite tracing and analysis in fluorescence microscopy images. Cytometry A 58:167-176.

Nelson R, Famiglietti Jr EV, Kolb H (1978) Intracellular staining reveals different levels of stratification for on- and off-center ganglion cells in cat retina. J Neurophysiol 41:472-483.

Nixon RA, Lewis SE, Dahl D, Marotta CA, Drager UC (1989) Early posttranslational modifications of the three neurofilament subunits in mouse retinal ganglion cells: neuronal sites and time course in relation to subunit polymerization and axonal transport. Brain Res Mol Brain Res 5:93-108.

Pollock GS, Vernon E, Forbes ME, Yan Q, Ma YT, Hsieh T, Robichon R, Frost DO, Johnson JE (2001) Effects of early visual experience and diurnal rhythms on BDNF mRNA and protein levels in the visual system, hippocampus, and cerebellum. J Neurosci 21:3923-3931.

Pollock GS, Robichon R, Boyd KA, Kerkel KA, Kramer M, Lyles J, Ambalavanar R, Khan A, Kaplan DR, Williams RW, Frost DO (2003) TrkB receptor signaling regulates developmental death dynamics, but not final number, of retinal ganglion cells. J Neurosci 23:10137-10145.

Poo MM (2001) Neurotrophins as synaptic modulators. Nat Rev Neurosci 2:24-32.

Rohrer B, Korenbrot JI, LaVail MM, Reichardt LF, Xu B (1999) Role of neurotrophin receptor TrkB in the maturation of rod photoreceptors and establishment of synaptic transmission to the inner retina. J Neurosci 19:8919-8930.

Seki M, Nawa H, Fukuchi T, Abe H, Takei N (2003) BDNF is upregulated by postnatal development and visual experience: quantitative and immunohistochemical analyses of BDNF in the rat retina. Invest Ophthalmol Vis Sci 44:3211-3218.

Sernagor E, Eglen SJ, Wong RO (2001) Development of retinal ganglion cell structure and function. Prog Retin Eye Res 20:139-174.

Sherry DM, Wang MM, Bates J, Frishman LJ (2003) Expression of vesicular glutamate transporter 1 in the mouse retina reveals temporal ordering in 
development of rod vs. cone and ON vs. OFF circuits. J Comp Neurol 465:480-498

Sun W, Li N, HeS (2002) Large-scale morphological survey of mouse retinal ganglion cells. J Comp Neurol 451:115-126.

Tagawa Y, Sawai H, Ueda Y, Tauchi M, Nakanishi S (1999) Immunohistological studies of metabotropic glutamate receptor subtype 6-deficient mice show no abnormality of retinal cell organization and ganglion cell maturation. J Neurosci 19:2568-2579.

Tian N, Copenhagen DR (2003) Visual stimulation is required for refinement of ON and OFF pathways in postnatal retina. Neuron 39:85-96.

Tolwani RJ, Buckmaster PS, Varma S, Cosgaya JM, Wu Y, Suri C, Shooter EM (2002) BDNF overexpression increases dendrite complexity in hippocampal dentate gyrus. Neuroscience 114:795-805.

Viegi A, Cotrufo T, Berardi N, Mascia L, Maffei L (2002) Effects of dark rearing on phosphorylation of neurotrophin Trk receptors. Eur J Neurosci 16:1925-1930.

Witkovsky P (2004) Dopamine and retinal function. Doc Ophthalmol 108:17-40.
Wong RO (1990) Differential growth and remodelling of ganglion cell dendrites in the postnatal rabbit retina. J Comp Neurol 294:109-132.

Woo NH, Teng HK, Siao CJ, Chiaruttini C, Pang PT, Milner TA, Hempstead BL, Lu B (2005) Activation of p75NTR by proBDNF facilitates hippocampal long-term depression. Nat Neurosci 8:1069-1077.

Xu B, Zang K, Ruff NL, Zhang YA, McConnell SK, Stryker MP, Reichardt LF (2000) Cortical degeneration in the absence of neurotrophin signaling: dendritic retraction and neuronal loss after removal of the receptor TrkB. Neuron 26:233-245.

Young RW (1984) Cell death during differentiation of the retina in the mouse. J Comp Neurol 229:362-373.

Zagrebelsky M, Holz A, Dechant G, Barde YA, Bonhoeffer T, Korte M (2005) The p75 neurotrophin receptor negatively modulates dendrite complexity and spine density in hippocampal neurons. J Neurosci 25:9989-9999.

Zheng JJ, Lee S, Zhou ZJ (2004) A developmental switch in the excitability and function of the starburst network in the mammalian retina. Neuron 44:851-864. 